\title{
A NOTE ON PRODUCTS OF HOMOGENEOUS TORSION FREE ABELIAN GROUPS
}

\author{
ROSS A. BEAUMONT
}

In his book on abelian groups, L. Fuchs remarks $[1$, p. 173] that a direct product of torsion free groups of rank one and the same type $\tau$ is a homogeneous torsion free group of type $\tau$. It is easy to give an example to show that this is not the case. Moreover, the example suggests the correct statement concerning the type set of an infinite product of homogeneous torsion free groups of the same type.

Let $H$ be the subgroup of the additive rationals $Q$ consisting of the rationals with square free denominator, and let $G=\prod_{p \in \pi} H_{p}$, where $\pi$ is the set of all primes, and $H_{p}=H$ for each prime $p \in \pi$. That is, $G$ is the direct product of a countably infinite number of copies of $H$, indexed by $\pi$. The type $\tau$ of $H$ is the type of the characteristic $\chi$ such that $\chi(p)=1$ for all $p \in \pi$. Since $H$ is a direct summand of $G, G$ contains elements of type $\tau$. For each prime $p \in \pi, 1 / p \in H$ and $h_{p}^{H}(1 / p)=0$ (where $h_{p}^{H}(x)$ is the $p$-height of $x \in H$ ). Let $u$ be the element of $G$ such that the $p$ th component of $u$ is $1 / p$. Since the $p$-height of $u$ in $G$ is no greater than the $p$-height of any one of its components in $H$, it follows that $h_{p}^{G}(u)=0$ for all $p \in \pi$. Thus, if $\chi(u)$ is the characteristic of $u$ in $G, \chi(u)$ is not equivalent to $\chi$, and the type of $u$ is not equal to $\tau$. Hence $H$ is not homogeneous.

We recall that a characteristic $\chi$ is nonnil if $0<\chi(p)<\infty$ for only a finite number of primes $p$. The type of a nonnil characteristic is called a nonnil type. Thus, if $\tau$ is a nonnil type, $\tau$ is the type of a characteristic such that $\chi(p)=0$ or $\chi(p)=\infty$ for all primes $p \in \pi$. Let $\tau$ be the type of a characteristic $\chi$. The underlying nonnil type of $\tau$ is the type $\mu$ of a nonnil characteristic $\Phi$ such that $\Phi(p)=\infty$ if and only if $\chi(p)=\infty$.

We remark that a type $\tau$ is nonnil if and only if $\tau$ is the type of a subring of $Q$. Moreover, if $A$ is a rank one group having type $\tau$, then the underlying nonnil type of $\tau$ is the type of $\operatorname{Hom}(A, A)$.

The set of types of the nonzero elements of a torsion free group $G$ is denoted by $T(G)$.

Theorem. Let $A$ be an infinite index set and let $\left\{G_{\alpha} \mid \alpha \in A\right\}$ be a collection of homogeneous torsion free groups of type $\tau$. Let $G=\prod_{\alpha \in A} G_{\alpha}$. Then $T(G)$ is the set of all types $\nu$ such that $\mu \leqq \nu \leqq \tau$, where $\mu$ is the underlying nonnil type of $\tau$.

Received by the editors November 20, 1968. 
Proof. Let $u$ be a nonzero element of $G$ and let $g_{\alpha} \in G_{\alpha}$ be the $\alpha$ th component of $u$. Then

$$
h_{p}^{G}(u)=\min \left\{h_{p}^{G}\left(g_{\alpha}\right) \mid \alpha \in A\right\},
$$

for each prime $p \in \pi$. Let $\tau$ be the type of a characteristic $\chi$. Since each $G_{\alpha}$ is homogeneous of type $\tau$, it follows that if $g_{\alpha} \neq 0$, the characteristic of $g_{\alpha}$ in $G_{\alpha}$ is equivalent to $\chi$. That is, for every $\alpha, h_{p}^{G \alpha}\left(g_{\alpha}\right)=\chi(p)$ for all but a finite number of primes $p$ and $h_{p}^{G_{\alpha}}\left(g_{\alpha}\right)=\infty$ if and only if $\chi(p)=\infty$. Therefore, $h_{p}^{G}(u) \leqq \chi(p)$ for all but a finite number of primes $p$ and $h_{p}^{G}(u)=\infty$ if and only if $\chi(p)=\infty$. Clearly, $h_{p}^{G}(u) \geqq \Phi(p)$ for all but a finite number of primes $p$, where $\Phi$ is a characteristic of the underlying nonnil type $\mu$ of $\tau$. Thus, if $\nu$ is the type of $u$ in $G$, $\mu \leqq \nu \leqq \tau$.

Now suppose that $\nu$ is any type such that $\mu \leqq \nu \leqq \tau$. Let $\mu, \nu$, and $\tau$ be represented by characteristics $\Phi, \psi$, and $\chi$ such that $\Phi \leqq \psi \leqq \chi$. Let $\pi_{1}=\{p \in \pi \mid \psi(p)=\chi(p)\}$. Then $\pi_{1} \supseteq\{p \in \pi \mid \Phi(p)=\psi(p)=\chi(p)=\infty\}$. Let $\pi_{2}=\{p \in \pi \mid \psi(p)<\chi(p)\}$. Hence, if $p \in \pi_{2}, \chi(p)<\infty$. For each $\alpha \in A$, there is an element $x_{\alpha} \in G_{\alpha}$ such that $h_{p}^{G}\left(x_{\alpha}\right)=\chi(p)$ for all $p \in \pi$. For each $p \in \pi_{2}$, define $x_{\alpha, p} \in G_{\alpha}$ by $x_{\alpha}=p^{\chi(p)-\psi(p)} x_{\alpha, p}$. Then $h_{p}^{G_{\alpha}}\left(x_{\alpha, p}\right)=\psi(p)$ and $h_{\alpha}^{G_{\alpha}}\left(x_{\alpha, p}\right)=\chi(g)$ for $q \neq p$. Partition the infinite set $A$ into subsets $B$ and $C$ such that $|B|=\left|\pi_{2}\right|$. Then $G \cong G_{1} \oplus G_{2}$, where $G_{1}=\prod_{\beta \in B} G_{\beta}=\prod_{p \in \pi_{2}} G_{\beta(p)}$ and $G_{2}=\prod_{\gamma \in C} G_{\gamma}$. Let $u$ be the element of $G_{1}$ which has the element $x_{\beta(p), p}$ as $\beta(p)$ th component. Since $h_{p}^{G_{\beta(p)}}\left(x_{\beta(p), p}\right)=\psi(p)$ and $h_{p}^{G_{\beta(p)}}\left(x_{\beta(p), p}\right)=\psi(q)$ if $q \neq p$, it follows that $h_{p}^{G 1}(u)=\psi(p)$ if $p \in \pi_{2}$ and $h_{p}^{G_{1}}(u)=\chi(p)=\psi(p)$ if $p \in \pi_{1}$. Thus, the type of $u$ in $G_{1}$ is $\nu$. Since $G_{1}$ is a direct summand of $G$, the type of $u$ in $G$ is $\nu$. Hence $G$ contains an element of type $\nu$ for every $\nu$ such that $\mu \leqq \nu \leqq \tau$. Combining this result with that of the first paragraph of the proof completes the proof of the theorem.

Corollary 1. Let $G=\prod_{\alpha \in A} G_{\alpha}$ as described in the above theorem. Then $G$ is homogeneous of type $\tau$ if and only if $\tau$ is nonnil. Moreover if $\tau$ is not nonnil, $|T(G)|=2^{\aleph_{0}}$.

Proof. If $\tau$ is nonnil, then $\mu=\tau$ where $\mu$ is the underlying nonnil type of $\tau$. By the theorem, $T(G)=\{\nu \mid \mu \leqq \nu \leqq \tau\}$. Hence $T(G)=\{\tau\}$, so that $G$ is homogeneous of type $\tau$. If $\tau$ is not nonnil, then $\tau$ is the type of a characteristic $\chi$ such that $0<\chi(p)<\infty$ for infinitely many primes $p$. Let $\pi^{\prime}=\{p \in \pi \mid 0<\chi(p)<\infty\}$. If $\Phi$ is a characteristic of the underlying nonnil type $\mu$ of $\tau, \Phi(p)=0$ for infinitely many primes $p \in \pi^{\prime}$. Thus, there are $2^{\aleph_{0}}$ distinct characteristics $\psi$ such that $\Phi \leqq \psi \leqq \chi$. It follows that there are $2^{\aleph_{0}}$ distinct types $\nu$ such that 
$\mu \leqq \nu \leqq \tau$, since there are only a countable infinity of characteristics in each type. By the theorem, $|T(G)|=2^{\aleph_{0}}$.

Another consequence of the Theorem is a uniqueness statement concerning the representation of a torsion free group as a product of homogeneous groups of the same type.

Corollary 2. Let $G=\prod_{\alpha \in A} G_{\alpha}=\prod_{\beta \in B} H_{\beta}$, where the $G_{\alpha}$ are homogeneous of type $\tau$ and the $H_{\beta}$ are homogeneous of type $\tau^{\prime}$. Then $\tau=\tau^{\prime}$.

Proof. If $A$ is finite, then $G$ is homogeneous of type $\tau$, and since $H_{\beta}$ is a direct summand of $G$ for each $\beta \in B, H_{\beta}$ is homogeneous of type $\tau$ for each $\beta \in B$. Thus, in this case $\tau=\tau^{\prime}$, and we may assume that $A$ is infinite. Since $\tau^{\prime} \in T(G)$, it follows from the theorem that $\mu \leqq \tau^{\prime} \leqq \tau$, where $\mu$ is the underlying nonnil type of $\tau$. Thus, $\tau=\tau^{\prime}$ if $\tau$ is nonnil. Assume that $\tau$ is not nonnil. Then it follows from Corollary 1 that $G$ is not homogeneous. Therefore $B$ is infinite, for otherwise $G=\prod_{\beta \in B} H_{\beta}$ would be homogeneous of type $\tau^{\prime}$. By the theorem, $T(G)=\left\{\nu \mid \mu \leqq \nu \leqq \tau^{\prime}\right\}$ where $\mu$ is the underlying nonnil type of $\tau^{\prime}$. Hence $\tau \leqq \tau^{\prime}$, and therefore $\tau=\tau^{\prime}$.

As a final application, suppose that $G$ is a homogeneous torsion free group of nonnil type $\tau$. Then the set of all mappings of $G$ into $G$ is an abelian group $\operatorname{Map}(G, G)$ and

$$
\operatorname{Map}(G, G) \cong \prod_{\boldsymbol{o} \in G} G_{\boldsymbol{g}}
$$

where $G_{g} \cong G$ for all $g \in G$. That is, $\operatorname{Map}(G, G)$ is an infinite product of copies of $G$. By Corollary 1, $\operatorname{Map}(G, G)$ is homogeneous of type $\tau$. Suppose that $H$ is any group such that $\operatorname{Map}(G, G) \cong \operatorname{Map}(H, H)$. Then $\operatorname{Map}(H, H)$ is a homogeneous torsion free group of type $\tau$. Moreover, $\operatorname{Map}(H, H) \cong \prod_{x \in H} H_{x}$, where $H_{x} \cong H$ for all $x \in H$. Thus, $H$ is a direct summand of $\operatorname{Map}(H, H)$, so that $H$ is a homogeneous torsion free group of type $\tau$.

\section{REFERENCE}

1. L. Fuchs, Abelian groups, Akadémiai Kiad6, Budapest, 1958.

UNIVERSITY OF WASHINGTON 